\title{
Investigation of the Mechanical Strength of Cell-plastics Fabricated Using Unicellular Green Algal Cells and Varying Weight Ratios of Biodegradable Polybutylene Succinate
}

\author{
Akihito Nakanishi ${ }^{1,2,{ }^{*}, \dagger}$, Kohei Iritani ${ }^{3, \dagger}$, Yuri Sakihama ${ }^{4}$, Marina Watanabe ${ }^{2}$ \\ ${ }^{1}$ Graduate School of Bionics, Tokyo University of Technology, Tokyo, Japan \\ ${ }^{2}$ School of Bioscience and Biotechnology, Tokyo University of Technology, Tokyo, Japan \\ ${ }^{3}$ School of Engineering, Tokyo University of Technology, Tokyo, Japan \\ ${ }^{4}$ Tokyo University of Technology, Tokyo, Japan \\ Email address: \\ nakanishiah@stf.teu.ac.jp (A. Nakanishi), iritanikh@stf.teu.ac.jp (K. Iritani), re19001b6@edu.teu.ac.jp (Y. Sakihama), \\ b01173031e@edu.teu.ac.jp (M. Watanabe) \\ ${ }^{*}$ Corresponding author \\ $\dagger$ Akihito Nakanishi and Kohei Iritani are co-first authors.
}

\section{To cite this article:}

Akihito Nakanishi, Kohei Iritani, Yuri Sakihama, Marina Watanabe. Investigation of the Mechanical Strength of Cell-plastics Fabricated Using Unicellular Green Algal Cells and Varying Weight Ratios of Biodegradable Polybutylene Succinate. International Journal of Microbiology and Biotechnology. Vol. 5, No. 4, 2020, pp. 159-164. doi: 10.11648/j.ijmb.20200504.11

Received: October 14, 2020; Accepted: October 23, 2020; Published: November 11, 2020

\begin{abstract}
Petroleum-derived plastics are currently indispensable; however, they are non-recyclable and exhaustible. Despite global progress in the research and development for biodegradable green plastics using recyclable resources, green plastics are unable to replace petroleum-derived plastics so far because of the costs involved in the extraction and purification processes. Due to the increasing demand for the development of innovative green plastics, cell-plastic composed of the green alga Chlamydomonas reinhardtii as an ingredient and biodegradable compounds as fillers was proposed as a novel green plastic. For a carbon-recycling system in the future, $C$. reinhardtii is advantageous due to higher growth activity using $\mathrm{CO}_{2}$ gas in atmosphere compared to other terrestrial plants. In addition, the rigidity of its cell wall would prevent the reduction of a mechanical strength of the cell-plastics. Green plastics made of organic polymers are expensive because of high producing cost. On the other hand, the plastics made of green algae, instead of organic polymers, would be expected reducing the price. Thus, the purpose of this study was to clarify whether the cell-plastics produced by substituting organic polymers with algal cells could perform mechanical and physical properties similar to existing plastics products. As a filler for cell-plastics, biodegradable polybutylene succinate (PBS), which form a self-standing film, was chosen in this study. PBS cell-plastics were examined for their following mechanical and physical properties: Young's modulus, tensile strength, thermal analysis, and surface hydrophilicity. PBS cell-plastic (1:1) composed of 50\% PBS ( $w / w)$ exhibited the potential to replace petroleum-derived plastics such as low-density polyethylene and polyvinyl chloride. This study introduces the practical possibility of PBS cell-plastics as green plastics.
\end{abstract}

Keywords: Cell-plastics, Green Alga, Chlamydomonas reinhardtii, Polybutylene Succinate (PBS), Biodegradable

\section{Introduction}

Petroleum-derived plastics such as polyethylene and polyvinyl chloride (PVC) are essential in the current society because of their low production cost, simple processing, and easy endowing strength for various purposes [1]. Petroleum-derived plastics, however, face several problems: finiteness of crude oil $[2,3]$; emission of unrecyclable $\mathrm{CO}_{2}$ into the atmosphere by disposal of plastic waste [4]; persistent plastics polluting terrestrial and oceanic environments [5]. Therefore, research and development of biodegradable green plastics as a carbon-recycling resource are ongoing worldwide [6, 7]. Although many green plastics, such as polylactic acid (PLA), have been proposed and produced so far, they cannot replace petroleum-derived 
plastics because of the high costs of extraction and production $[8,9]$. Therefore, development of methods with novel ideas to efficiently produce green plastics is required.

Recyclable cell-plastics were previously suggested and produced using a unicellular green alga Chlamydomonas reinhardtii as an ingredient [10]. C. reinhardtii as a $\mathrm{CO}_{2}$-assimilating species is a carbon-recycling resource [11], and it is more advantageous due to higher growth activity than that of popular terrestrial plants and the ability to produce value-added chemicals, such as lipids and pigments [11-13]. However, C. reinhardtii has a rigid cell wall [14] and this rigidity is its demerit in extracting intracellular products during material production. On the other hand, the rigidity could be a merit when used as a raw material possessing mechanical strength. The concept of cell-plastic suggests that the plastic be composed of $C$. reinhardtii cells as the rigid component. These cells are weakly bound with noncovalent bonds, so the macro molding would not be inhibited even while using these rigid cells as the components; however, its mechanical strength as a plastic material would be diminished. To overcome this issue, glycerol and bovine serum albumin were used as intercellular fillers to connect each cell and a thin organic layer was applied as a reinforcer to coat the laminate structures of the cell-layers, resulting in the formation of a self-supporting film of cell-plastic. Although cell-plastic had the potential to be the new green plastic, it was problematic owing to its time-consuming and complicated process since its production required almost complete water removal and layer laminations. These problems could be attributable to the intercellular filler glycerol that prevented the drying of the cell-plastic film due to its hydrophilic nature. Additionally, although glycerol could be correlatedly connected to hydrophilic cells, the use of glycerol decreased the mechanical strength of the cell-plastics compared to the strength of ordinal petroleum-derived plastics since the cells were connected with a weak bond. Therefore, an appropriate intercellular filler, with simple molding feasibility and the mechanical strength for a self-supporting film by itself, is needed to compose the cell-plastics. In this study, biodegradable polybutylene succinate (PBS) was used as the intercellular filler (Figure 1). PBS has mechanical strength properties similar to those of low-density polyethylene (LDPE) and high-density polyethylene (HDPE), making PBS suitable for food packaging and agricultural substances [15]. Additionally, composite materials of PBS with lignin derivatives [16] and organic clays [17] have been developed to improve its mechanical strength and thermal tolerance. When lignin derivatives are used, incorporation of a hydrogen bond donor/acceptor site into lignin causes formation of hydrogen bonds with the carbonyl and terminal hydroxy groups of PBS, leading to improved mechanical strength. Since the $C$. reinhardtii cells used in this study have many hydroxy groups on their cell walls, it was theorized that hydrogen bonding could occur between the hydroxy groups on the cell walls and the carbonyl groups on PBS, resulting in the formation of a unified PBS cell-plastic. Extraction and purification costs of PBS are higher than that of preparation of $C$. reinhardtii cells.
Therefore, decreasing the ratio of PBS in the green plastic composition by using $C$. reinhardtii cells could greatly contribute to reducing the cost of producing cell-plastics, making PBS cell-plastics a superior green plastic.

In this study, PBS cell-plastics possessing varying ratios of cells and PBS were prepared. After analyzing their surface structures and film thicknesses using a scanning electron microscope (SEM), the mechanical properties of Young's modulus and tensile strength and physical properties of differential thermal fastness and contact angle were evaluated. Additionally, the properties of PBS cell-plastics and existing petroleum-derived plastics were compared and considering the possibility of substitution, the practicality of PBS cell-plastics was also predicted. Our study suggests that PBS cell-plastics could be a novel green plastic.

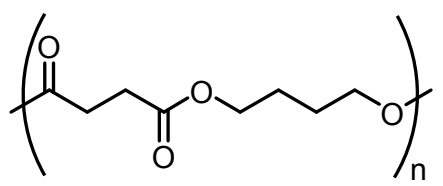

Figure 1. Chemical structure of polybutylene succinate (PBS).

\section{Materials and Methods}

\subsection{Culturing Conditions}

C. reinhardtii strain C-8: NIES-2239 was cultured under a white-fluorescent light intensity of $50 \mu \mathrm{mol}$ photons $\cdot \mathrm{m}^{-2} \cdot \mathrm{s}^{-1}$ at $23^{\circ} \mathrm{C}$ in modified Bold (MB) $6 \mathrm{~N}$ medium, as described in a previous study [18]. Cell culture was started with a concentration of $1.0-2.0 \times 10^{6}$ cells $\cdot \mathrm{L}^{-1}$, in which cells were obtained from a pre-culture in $\mathrm{MB} 6 \mathrm{~N}$ medium to avoid nitrogen starvation. The aeration rate of $0.8 \% \mathrm{CO}_{2}$ was 0.1 vvm and controlled with a flow meter (Model RK1200 series, KOFLOC, Kyoto, Japan).

\subsection{Preparation of Cell Plastics}

Cell plastics comprised a mixture of $C$. reinhardtii cells and PBS. Cell culture was centrifuged at $5000 \times g$ for 1 min at $23^{\circ} \mathrm{C}$; the supernatant was discarded and the harvested cells were collected as the precipitate. After vortexing the harvested cells with distilled water, the mixture was centrifuged at $5000 \times \mathrm{g}$ for $1 \mathrm{~min}$ at $23^{\circ} \mathrm{C}$ to collect the washed cells. The washed cells were air-dried for $16 \mathrm{~h}$ and dispersed using PowerMasher ${ }^{\circledR}$ II and BioMasher ${ }^{(B)} \mathrm{V}$ (KANTO KAGAKU, Ibaraki, Japan). After dispersing, the cells were used as ingredients of cell-plastics. PBS to be used as filler was prepared by dissolving a tablet of PBS (14-18 mg per tablet, Mitsubishi Chemical Corporation, Tokyo, Japan) in $1 \mathrm{~mL}$ of dichloromethane (FUJIFILM Wako Pure Chemical Corporation, Osaka, Japan). The dispersed cells were added to the PBS solution maintaining the ratio of the weight of cells and PBS as $1: 1,2: 1,3: 1,5: 1$, and 10:1, after which these mixtures were dried and molded as cell-plastics on a baking tray. The PBS cell-plastics composed of these mixing ratios were finally named as cell-plastic (1:1), cell-plastic $(2: 1)$, 
cell-plastic (3:1), cell-plastic (5:1), and cell-plastic (10:1).

\subsection{Electron Microscopy}

PBS cell-plastics and PBS were cut into $5 \mathrm{~mm}^{2}$ squares and coated with $\mathrm{Au}$ molecules using the ion coater IB-2 (EIKO Corporation, Ibaraki, Japan) to obtain SEM images. After coating, images of each cell-plastic were taken using an SEM (JSM-6060LV; Japan Electron Optics Laboratory Co., Ltd., Tokyo, Japan). To evaluate the film thickness, three points of each of the three films of PBS cell-plastics and PBS were measured, and averages and standard deviations were calculated.

\subsection{Evaluation of Young's Modulus and Tensile Strength}

The cell-plastics were analyzed to evaluate the Young's modulus and tensile strength using a tensile strength tester (TesTex, Zurich, Switzerland). The cell-plastics were cut into rectangles $(5 \mathrm{~mm} \times 7.5 \mathrm{~mm})$ and a crosshead rate of 1.00 $\mathrm{mm} \cdot \mathrm{min}^{-1}$ was maintained. Following the test, a load-displacement curve was plotted. To calculate the Young's modulus and tensile strength, a stress-strain curve was plotted by dividing the load and displacement by the cross-section and the initial length of the test piece, respectively. The cross-section was calculated as the product of the width $(5$ $\mathrm{mm}$ ) and average thickness, which were obtained from the SEM observations of the small sample pieces cut using scissors. Young's modulus was derived using the slope of an initial straight line approximated via the least squares method. The tensile strength was defined as the maximum stress value. The Young's modulus and tensile strength of each film were the averages of 9 test pieces obtained by cutting three different films into three different-sized areas.

\subsection{Differential Thermal Analyses}

Differential thermal analysis (DTA) was carried out using the DTA-60 instrument (Shimadzu Corporation, Kyoto, Japan) at a heating rate of $5^{\circ} \mathrm{C} \cdot \mathrm{min}^{-1}$ under nitrogen gas $\left(100 \mathrm{~mL} \cdot \mathrm{min}^{-1}\right)$.

\subsection{Evaluation of the Contact Angles}

Drop Master 300 (Kyowa Interface Science Co., Ltd., Saitama, Japan) was used to evaluate the contact angle. The contact angle was determined after depositing a drop of water on the films.

\section{Results and Discussion}

\subsection{Composition of the PBS Cell-plastics}

Dried cells of $C$. reinhardtii strain C-8: NIES-2239 were used as ingredients of the cell-plastics. These cells were prepared as diffused ingredients using PowerMasher ${ }^{\circledR}$ II equipped with BioMasher ${ }^{\mathrm{B}} \mathrm{V}$ because the cultured cells could have aggregated after washing and drying. Although the PowerMasher ${ }^{\circledR}$ II equipped with BioMasher ${ }^{\circledR} \mathrm{V}$ used for cell diffusion is a tool to break tender cells, it could not crush $C$. reinhardtii cells, demonstrating that the cells retained their original shapes after diffusion treatment, as observed under an optical microscope (data not shown). The extraction of intracellular contents like lipids from Chlamydomonas sp., including $C$. reinhardtii, generally requires a powerful tool such as a bead beater to break the cells [18-19], implying that the $C$. reinhardtii cells used in this study could maintain their cell shapes even after diffusion treatment with the PowerMasher ${ }^{(B)}$ II equipped with BioMasher ${ }^{\circledR} \mathrm{V}$.

In this study, all PBS cell-plastics were self-standing films, despite $C$. reinhardtii cells not forming films but only green powders after drying treatment (Figure 2). To produce the cell-plastics, C. reinhardtii cells and PBS were used as the green ingredient and white filler, respectively, resulting in the color of the PBS plastic being green to deep green, as the proportion of cells increased. Random-sized cell aggregations were detected upon visual examination as dotted green spots on PBS cell-plastics even though the cell size was approximately 5 $\mu \mathrm{m}$. This indicated that the aggregations were formed as partial clusters without spreading the cells in the PBS cell-plastics evenly. Additionally, the PBS cell-plastics tended to be rougher structures as the ratio of PBS decreased. Furthermore, cell-plastic (5:1) and cell-plastic (10:1) were visually porous structures. The porous structures could be attributed to the insufficient amount of PBS to fill the intercellular matrix.

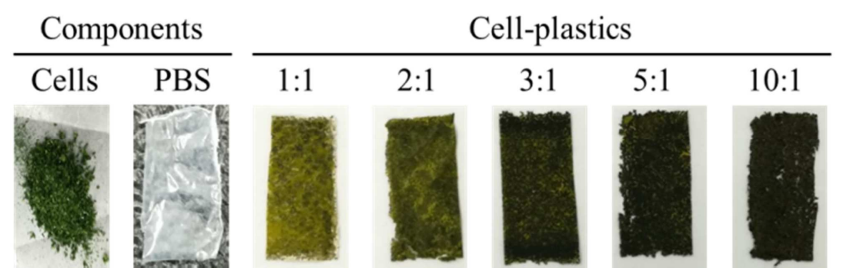

Figure 2. Pictures of cell-plastic components (cells and PBS) and PBS cell-plastics. Mixing ratios (w:w) between cells and PBS were shown in cell plastics column.

\subsection{Analysis of Mechanical and Physical Properties}

The surfaces and cross-sections of the PBS cell-plastics and PBS were analyzed with an SEM to inspect the structures in detail (Figure 3). PBS film without cells showed a homogeneous surface, whereas cell-plastic (1:1) and cell-plastic (2:1) exhibited heterogeneous surfaces possibly due to cells without porous structures. Cell-plastic (3:1), cell-plastic (5:1), and cell-plastic (10:1) displayed porous structures on their surfaces, presumably attributable to the increasing ratios of cells. Analysis of the SEM images of the cross-sections revealed that not only the surfaces but also the insides of cell-plastic (3:1), cell-plastic (5:1), and cell-plastic (10:1) presented porous structures owing to the low ratios of PBS, implying that PBS was insufficient as a filler and could not coat and connect each cell as a component. After measuring the thicknesses of the PBS cell-plastics and PBS in the SEM images, the averages and standard deviations were calculated for the analysis of the mechanical properties of the films (Table 1). The thickness of the PBS film was $12 \pm 2 \mu \mathrm{m}$, and those of PBS cell-plastics gradually increased to $72 \pm 12$ $\mu \mathrm{m}$ for cell-plastic (10:1), indicating that the films became thicker as the ratio of cells in the PBS cell-plastics increased. Aggregating structures, such as clusters without evenly 
diffused cells were formed, possibly contributing to the thickness of the film. Additionally, the standard deviation values increased as the ratio of the cells in the PBS cell-plastics increased. The results suggest that cell-plastics with a higher ratio of cells have a porous structure and that achieving uniform film thicknesses might be difficult.

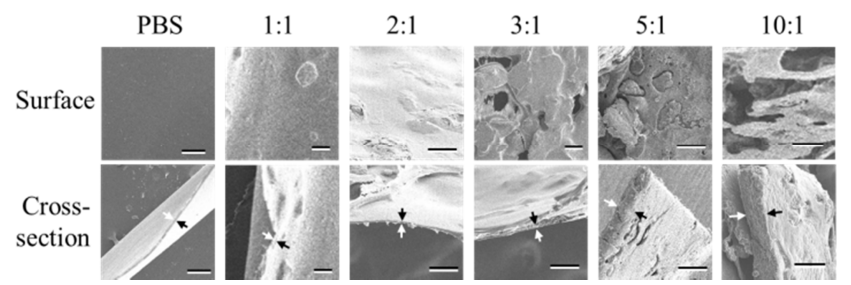

Figure 3. SEM images of PBS and PBS cell-plastics. Scale-bars were 100 $\mu m$. Regions characterized by arrows on each image of cross-section means plastic cross-section. Mixing ratios (w:w) between cells and PBS were shown above SEM images.

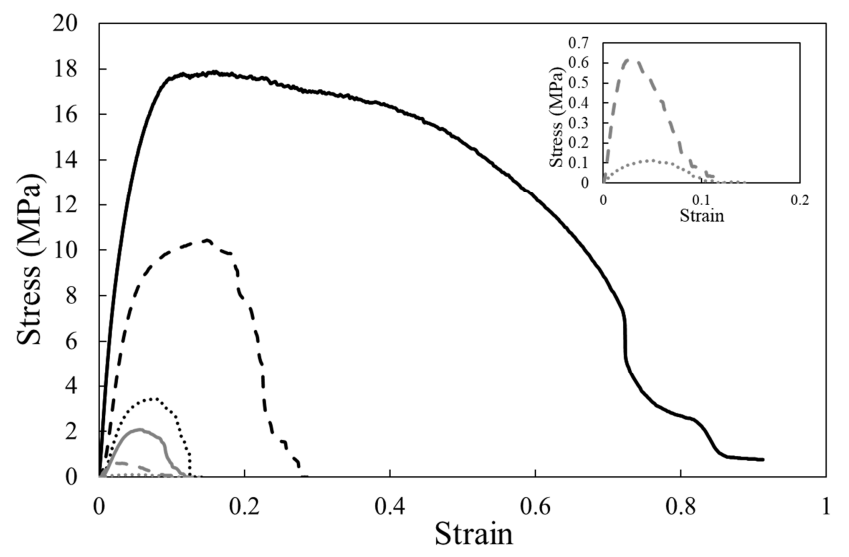

Figure 4. Typical stress-strain curves of PBS film (black solid line), films of cell-plastic (1:1) (black broken line), cell-plastic (2:1) (black dotted line), cell-plastic (3:1) (gray solid line), cell-plastic (5:1) (gray broken line), and cell-plastic (10:1) (gray dotted line). Those of cell-plastic (5:1) and cell-plastic (10:1) with the vertical axis adjusted are inserted.

A tensile strength test was performed to evaluate the mechanical properties of the fabricated films. Each typical stress-strain curve is shown in Figure 4, and the Young's modulus and tensile strength values are shown in detail in Table 2. Both, the Young's modulus and the tensile strength of the PBS film were the maximum, and the mechanical strength decreased with increasing ratios of cells in the PBS cell-plastics. A possible explanation for this is that the cells were not uniformly dispersed in the PBS film and partially formed aggregates, resulting in inefficient hydrogen bond formation between the cells and the PBS polymer. Additionally, the standard deviation was greater in cell-plastic (5:1) and cell-plastic (10:1). The local structures of the test pieces prepared by cutting could presumably influence the mechanical strength due to their heterogeneous structures. For practical application of cell-plastics, it is necessary to consider their mechanical strength and production cost. In terms of mechanical strength, PBS cell-plastics could be used instead of existing green plastics to replace petroleum-derived plastics as green plastics. Food packages, which comprise about $40 \%$ of the waste of petroleum-derived plastics, are mostly made of LDPE that has a Young's modulus of 165.3 MPa and a tensile strength of 10.3 MPa [20]. Among the prepared cell-plastics, cell-plastic (1:1) had a Young's modulus of $240 \pm 90 \mathrm{MPa}$ and a tensile strength of $8.8 \pm 3.8 \mathrm{MPa}$, comparable with those of LDPE. Furthermore, PVC, which is also used for manufacturing food packaging materials, has a Young's modulus of 4.8-12.4 MPa and a tensile strength of 5.5-26.2 $\mathrm{MPa}$ [20]. Although the Young's modulus value of cell-plastic $(1: 1)$ is considerably lower than that of flexible PVC, the tensile strength of the former competes with the latter, suggesting that there is a possibility of replacement of flexible PVC by this cell-plastic. Hence, cell-plastic (1:1) could potentially replace petroleum-derived plastics such as LDPE and PVC while using only half the amount of PBS. Moreover, production of PBS entails high costs for the extraction and purification of the polymer. Therefore, by using cells in PBS cell-plastics, cost reduction can be achieved since cells self-propagate via photosynthesis. Cell-plastic (1:1) has potential for practical bioplastic application.

Table 1. Thickness of PBS cell-plastics and PBS.

\begin{tabular}{lllllll}
\hline Cell: PBS $(\boldsymbol{w} / \boldsymbol{w})$ & $\mathbf{1 : 1}$ & $\mathbf{2 : 1}$ & $\mathbf{3 : 1}$ & $\mathbf{5 : 1}$ & $\mathbf{1 0 : 1}$ \\
\hline Thickness $(\mu \mathrm{m})$ & $19 \pm 2$ & $27 \pm 3$ & $36 \pm 4$ & $59 \pm 6$ & $72 \pm 12$ & $12 \pm 2$ \\
\hline
\end{tabular}

Mixing ratios $(w: w)$ between cells and PBS were shown in cell: PBS column. Values are the averages of three replicated experiments, $\pm \mathrm{SD}$.

Table 2. Summary of average values of Young's modulus and tensile strength of PBS film, films of cell-plastic (1:1), cell-plastic (2:1), cell-plastic (3:1), cell-plastic (5:1), and cell-plastic (10:1).

\begin{tabular}{lll}
\hline & Young's modulus (Mpa) & Tensile strength (Mpa) \\
\hline PBS film & $450 \pm 50$ & $25 \pm 6$ \\
Cell-plastic (1:1) & $240 \pm 90$ & $8.8 \pm 3.8$ \\
Cell-plastic (2:1) & $82 \pm 40$ & $3.3 \pm 0.5$ \\
Cell-plastic (3:1) & $60 \pm 17$ & $2.1 \pm 0.5$ \\
Cell-plastic (5:1) & $32 \pm 18$ & $0.66 \pm 0.39$ \\
Cell-plastic (10:1) & $6.3 \pm 7.1$ & $0.16 \pm 0.13$ \\
\hline
\end{tabular}

DTA was performed to evaluate the thermal properties of cell-plastic (1:1), PBS film, and C. reinhardtii cells (Figure 5). DTA of the PBS film revealed an endothermic peak at $115^{\circ} \mathrm{C}$ due to softening. Furthermore, a sharp decrease in mass was observed at approximately $350^{\circ} \mathrm{C}$, and the $10 \%$ weight loss temperature was noted to be $343^{\circ} \mathrm{C}$. In the case of $C$. reinhardtii cells alone, the mass gradually started decreasing at approximately $150^{\circ} \mathrm{C}$, and the $10 \%$ weight loss temperature was $316^{\circ} \mathrm{C}$, after which the cells were carbonized at higher temperatures. In cell-plastic (1:1), the mass started gradually decreasing at approximately $150^{\circ} \mathrm{C}$, with a sharp decrease at approximately $350^{\circ} \mathrm{C}$, similar to the PBS film, and the $10 \%$ weight loss temperature was $270^{\circ} \mathrm{C}$. Herein, a black substance 
that seemed to have eventually carbonized, was left (data not shown). The thermophysical properties of cell-plastic (1:1) were similar to those of PBS at room temperature, indicating the possibility of its use as a bioplastic. Therefore, cell-plastic (1:1) could be a substitute for LDPE and PVC, since it displays the physical properties of both PBS and green algal cells.

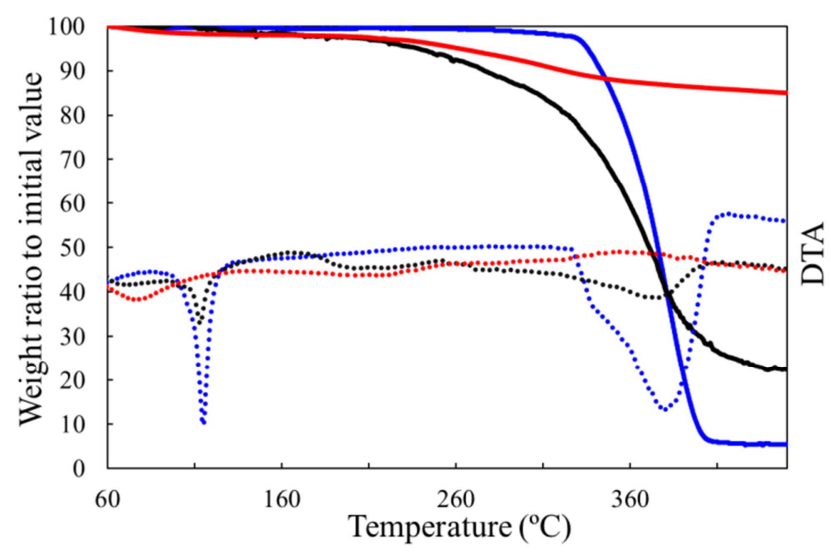

Figure 5. Thermogravimetric analyses (solid lines) and differential thermal analyses (dotted lines) of cell-plastic (1:1) (black), PBS film (blue), and cell (red).
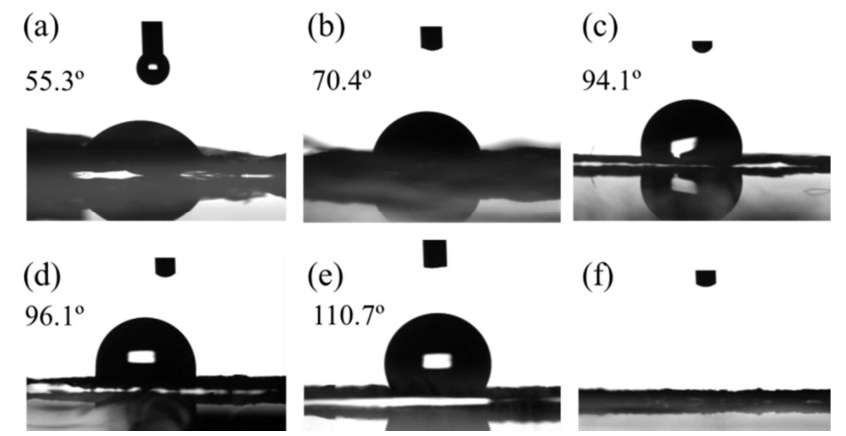

(f)

Figure 6. Side view images after dropping a water drop on films of (a) PBS (b) cell-plastic (1:1), (c) cell-plastic (2:1), (d) cell-plastic (3:1), (e) cell-plastic (5:1), and (f) cell-plastic (10:1). Inserted values show contact angles of each film.

The contact angles of the films were measured to investigate the hydrophilicity of their surfaces (Figure 6). When a drop of water was placed on each of the produced films, the contact angle was $55.3^{\circ}$ on the PBS film, and the angle increased along with the increase in the ratio of cells, up till cell-plastic (5:1). However, in the case of cell-plastic (5:1), after indicating a contact angle of $110.7^{\circ}$, the droplet was absorbed in a few minutes. The increase in contact angle could point towards the Lotus effect as per the increase in the proportion of the cell-exposed region on the surfaces of the films. On the other hand, in the case of cell-plastic (10:1), the droplet was immediately absorbed after being placed on the surface, suggesting that the absorption of water on porous structures supersedes the Lotus effect because of the high porosity of the film. Cell-plastic (1:1) demonstrated an appreciable degree of water repellency, suggesting the possibility of its use as a packaging material similar to LDPE and PVC.

\section{Conclusions}

PBS cell-plastics composed of $C$. reinhardtii cells and PBS were innovatively produced as composite resources using different mixing ratios. In this study, their mechanical properties, namely Young's modulus and tensile strength and physical properties, namely differential thermal fastness and contact angle were determined, after analyzing their SEM images. Cell-plastic (1:1), composed of only 50\% PBS, exhibited mechanical and physical properties comparable to those of LDPE and PVC, suggesting its applicability in packaging and its potential in replacing petroleum-based plastics. PBS cell-plastics are therefore, feasible as green plastics in the future.

\section{Abbreviation}

PBS, polybutylene succinate; $w / w$, weight/ weight; PLA, polylactic acid; LDPE, low-density polyethylene, HDPE, high-density polyethylene; SEM, scanning electron microscope; MB, modified Bold; DTA, differential thermal analysis; PE, polyethylene; PVC, polyvinyl chloride.

\section{Conflict of Interest}

The authors declare they have no competing interests.

\section{Acknowledgements}

The authors would like to thank Prof. Yoshihisa Tanaka for the technical support. This work was supported by Feasibility Study Program of the New Energy and Industrial Technology Development Organization (NEDO) (19101607-0). We appreciated sample provision and advices for this study given by Satoshi Kato in Mitsubishi Chemical Corporation and also supports by Editage (www.editage.com) for English language editing.

\section{References}

[1] Geyer R, Jambeck JR, Law KL (2017) Production, use, and fate of all plastics ever made. Sci. Adv. 3: e1700782.

[2] Shahbaz M, Nasreen S, Ling CH, Sbia R (2014) Causality between trade openness and energy consumption: what causes what in high, middle and low income countries. Energy Policy 70: $126-143$.

[3] Yao X, Yasmeen R, Padda IUH, Shah WUH, Kamal MA (2020) Inequalities by energy sources: an assessment of environmental quality. PLoS One 15: e0230503.

[4] Villanueva BR, Salvador MB, Huelgas RG (2019) Change climate and health. Rev. Clín. Esp. 219: 260-265.

[5] Barnes DKA, Galgani F, Thompson RC, Barlaz M (2009) Accumulation and fragmentation of plastic debris in global environments. Phil. Trans. R. Soc. B 364: 1985-1998. 
[6] Liu L, Fishman ML, Hicks KB, Liu CK (2005) Biodegradable composites from sugar beet pulp and poly (lactic acid). J. Agr. Food Ch. 53: 9017-9022.

[7] Sonseca A, Madani S, Rodríguez G, Hevilla V, Echeverría C, Fernández-García M, Muñoz-Bonilla A, Charef N, López D (2020) Multifunctional PLA blends containing chitosan mediated silver nanoparticles: Thermal, mechanical, antibacterial, and degradation properties. Nanomaterials 10: 22.

[8] Ammala A, Bateman S, Dean K, Petinakis E, Sangwan P, Wong S, Yuan Q, Yu L, Patrick C, Leong KH (2011) An overview of degradable and biodegradable polyolefins. Prog. Pol. Sci. 36: 1015-1049.

[9] Iles A, Martin AN (2013) Expanding bioplastics production: sustainable business innovation in the chemical industry. $J$. Cleaner Prod. 45: 38-49.

[10] Nakanishi A, Iritani K, Sakihama Y, Ozawa N, Mochizuki A, Watanabe M (2020) Construction of cell-plastics as neo-plastics consisted of cell-layer provided green alga Chlamydomonas reinhardtii covered by two-dimensional polymer. $A M B$ Expr. 10: 112.

[11] Hang LT, Mori K, Tanaka Y, Morikawa M, Toyama T (2020) Enhanced lipid productivity of Chlamydomonas reinhardtii with combination of $\mathrm{NaCl}$ and $\mathrm{CaCl}_{2}$ stresses. Bioproc. Biosyst. Eng. 43: 971-980.

[12] Sun H, Mao X, Wu T, Ren Y, Chen F, Liu B (2018) Novel insight of carotenoid and lipid biosynthesis and their roles in storage carbon metabolism in Chlamydomonas reinhardtii. Bioresour. Technol. 263: 450-457.

[13] Wang B, Li Y, Wu N, Lan CQ (2008) $\mathrm{CO}_{2}$ bio-mitigation using microalgae. Appl. Microbiol. Biotechnol. 79: 707-718.
[14] Hwang HJ, Kim YT, Kang NS, Han JW (2018) A simple method for removal of the Chlamydomonas reinhardtii cell wall using a commercially available subtilisin (alcalase). J. Mol. Microbiol. Biotechnol. 28: 169-178.

[15] Nazrin A, Sapuan SM, Zuhri MYM, Ilyas RA, Syafiq R, Sherwani SFK (2020) Nanocellulose reinforced thermoplastic starch (TPS), polylactic acid (PLA), and polybutylene succinate (PBS) for food packaging applications. Front Chem. 8: 213.

[16] Zhang Y, Zhou S, Fang X, Zhou X, Wang J, Bai F, Peng S (2019) Renewable and flexible UV-blocking film from poly (butylene succinate) and lignin. Eur. Polym. J. 116: 265-274.

[17] Shih YF, Wang TY, Jeng RJ, Wu JY, Teng CC (2007) Biodegradable nanocomposites based on poly (butylene succinate)/organoclay. J. Polym. Environ. 15: 151-158.

[18] Ho SH, Nakanishi A, Kato Y, Yamasaki H, Chang JS, Misawa N, Hirose Y, Minagawa J, Hasunuma T, Kondo A (2017) Dynamic metabolic profiling together with transcription analysis reveals salinity-induced starch-to-lipid biosynthesis in alga Chlamydomonas sp. JSC4. Sci. Rep. 7: 45471.

[19] Nakanishi A, Aikawa S, Ho SH, Chen CY, Chang JS, Hasunuma T, Kondo A (2014) Development of lipid productivities under different $\mathrm{CO}_{2}$ conditions of marine microalgae Chlamydomonas sp. JSC4. Bioresour. Technol. 152: 247-252.

[20] Brandrup J, Immergut EH, Grulke EA (1999) Polymer handbook, 4th edn. Wiley, New York, Chichester, Weinheim, Brisbane, Singapore, Toronto, pp V162-V165. 